ARTICLE

\title{
Adaptive introgression between Anopheles sibling species eliminates a major genomic island but not reproductive isolation
}

\author{
Chris S. Clarkson ${ }^{1, \star}$, David Weetman ${ }^{1, \star}$, John Essandoh ${ }^{1,2}$, Alexander E. Yawson $^{2,3}$, Gareth Maslen $^{4}$, \\ Magnus Manske ${ }^{4}$, Stuart G. Field ${ }^{5}$, Mark Webster ${ }^{6}$, Tiago Antão ${ }^{1}$, Bronwyn Maclnnis ${ }^{4}$, \\ Dominic Kwiatkowski ${ }^{4,7} \&$ Martin J. Donnelly ${ }^{1,4}$
}

\begin{abstract}
Adaptive introgression can provide novel genetic variation to fuel rapid evolutionary responses, though it may be counterbalanced by potential for detrimental disruption of the recipient genomic background. We examine the extent and impact of recent introgression of a strongly selected insecticide-resistance mutation (Vgsc-1014F) located within one of two exceptionally large genomic islands of divergence separating the Anopheles gambiae species pair. Here we show that transfer of the Vgsc mutation results in homogenization of the entire genomic island region ( $\sim 1.5 \%$ of the genome) between species. Despite this massive disruption, introgression is clearly adaptive with a dramatic rise in frequency of Vgsc-1014F and no discernable impact on subsequent reproductive isolation between species. Our results show (1) how resilience of genomes to massive introgression can permit rapid adaptive response to anthropogenic selection and (2) that even extreme prominence of genomic islands of divergence can be an unreliable indicator of importance in speciation.
\end{abstract}

\footnotetext{
${ }^{1}$ Department of Vector Biology, Liverpool School of Tropical Medicine, Pembroke Place, Liverpool L3 5QA, UK. ${ }^{2}$ Cape Coast Department of Entomology and Wildlife, School of Biological Science, University of Cape Coast, Cape Coast, Ghana. ${ }^{3}$ Biotechnology and Nuclear Agriculture Research Institute, Ghana Atomic Energy Commission, PO Box LG 80, Legon, Accra, Ghana. ${ }^{4}$ Malaria Programme, Wellcome Trust Sanger Institute, Hinxton, Cambridge CB10 1RQ, UK. ${ }^{5}$ Department of Microbiology, Immunology and Pathology, Colorado State University, Fort Collins, Colorado 80523, USA. ${ }^{6} 18$ a Church Lane, Hornsey, London N8 7BU, UK. ${ }^{7}$ Wellcome Trust Centre for Human Genetics, University of Oxford, Oxford OX3 7BN, UK. * These authors contributed equally to this work. Correspondence and requests for materials should be addressed to C.S.C. (email: csc@liv.ac.uk).
} 
A nthropogenic habitat changes present a difficult evolutionary challenge for both intentionally and unintentionally targeted organisms, because of the speed at which they occur. Introgressive hybridization between incompletely reproductively isolated species provides a mechanism for the rapid acquisition of novel genetic variation which can accelerate adaptive evolution and is of recognized importance for plants ${ }^{1}$. However, only a few clear cases have been demonstrated in animals, for example, the transfer of rodenticide tolerance between mouse species ${ }^{2}$ and of wing colour patterns among Heliconius butterflies ${ }^{3,4}$. A major obstacle to adaptive introgression is the rate at which recombination can separate beneficial genetic variants within an introgressed fragment from the wider donor background. The disruptive effect of this perturbation of epistasis within the recipient species genome is likely to be deleterious ${ }^{5}$. This may be exacerbated if introgressed adaptive variants are located in low recombination regions, because the hitchhiked portions of the donor species' genome will take longer to eliminate. Furthermore, because low recombination regions often exhibit elevated interspecific differentiation ${ }^{6-8}$, disruption by potentially adaptive introgression may be particularly acute if divergent selection on variants in the region underpins differentiation. Finally, if species are very closely related and much of the interspecific divergence of their genomes is represented in low recombination regions, this detrimental effect of introgression might impact reproductive isolation directly. However, the association of differentiation with divergent selection is controversial. Low recombination regions are prone to enhanced drift, recurrent background selection and recurrent hitchhiking, which can generate similar patterns in the genome to those predicted under strong divergent selection ${ }^{7,9-11}$. Although usually very difficult in wild populations, recent anthropogenic selection allowed us to investigate the extent and impact of adaptive introgression into a major 'genomic island' region postulated to be involved in divergent selection between the Anopheles gambiae species pair ${ }^{12,13}$.
The $\mathrm{M}$ and $\mathrm{S}$ forms of $A$. gambiae are morphologically indistinguishable and were originally identified by fixed differences in ribosomal DNA near the centromere of the $\mathrm{X}$ chromosome ${ }^{14}$. Though recently elevated to species status as A. coluzzii ( $\mathrm{M}$ form) and A. gambiae sensu stricto ( $\mathrm{S}$ form $)^{15}$, for continuity with past work we retain the nomenclature of $\mathrm{M}$ and $\mathrm{S}$, but discuss how our results bear on this formal species definition. Divergence of $M$ and $S$ is thought to be driven by ecological niche separation of larval habitats ${ }^{16}$. Differences in swarming locations have also been documented ${ }^{17}$, and even in mixed swarms mating is usually assortative ${ }^{18}$. However, $\mathrm{M}$ and $\mathrm{S}$ lack postzygotic isolation in the laboratory ${ }^{19}$ and hybrids are found occasionally in wild populations, although this frequency varies with country ${ }^{20}$. Turner et al. ${ }^{12}$ identified two large regions of the genome towards the centromeres of chromosomes $\mathrm{X}$ and $2 \mathrm{~L}$ that exhibit exceptional divergence between $\mathrm{M}$ and $\mathrm{S}$ forms. This novel discovery provided evidence compatible with mosaic genome models of ecological speciation with gene flow ${ }^{21,22}$ and helped to spur the field of speciation genomics. Such 'genomic islands of divergence' are hypothesized to arise via selection acting on a small number of physically linked variants, and grow through hitchhiking of additional physically linked adaptive and neutral loci ${ }^{11,12,23-25}$. Moreover, although hybrids may be selected agains ${ }^{26}$, there is clear evidence for at least some contemporary gene flow extending beyond the $F_{1}$ generation throughout the range in which $M$ and $S$ co-occur ${ }^{26-28}$, a key assumption of mosaic genome models of ecological speciation ${ }^{7,22}$. Nevertheless, discovery of additional areas of genomic divergence ${ }^{29-31}$ supported theoretical concerns ${ }^{7,9}$ that the $2 \mathrm{~L}$ and $\mathrm{X}$ genomic islands might be unrelated to speciation, their size arising via recurrent background selection and hitchhiking in the areas of extremely low recombination. Resolution of these competing hypotheses has been hindered by the complexity of phenotypic differences between the species pair ${ }^{16}$, which make laboratory studies very difficult. As a consequence, the importance of large genomic islands in the speciation process remains unclear.

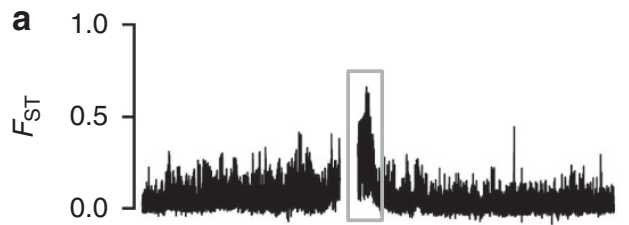

M-wt vs. S

b

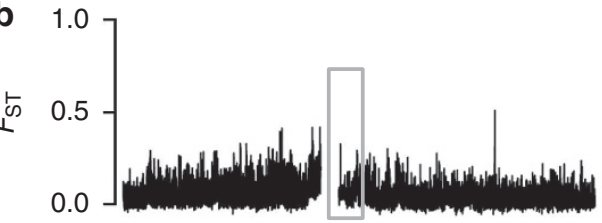

M-kdr vs. S
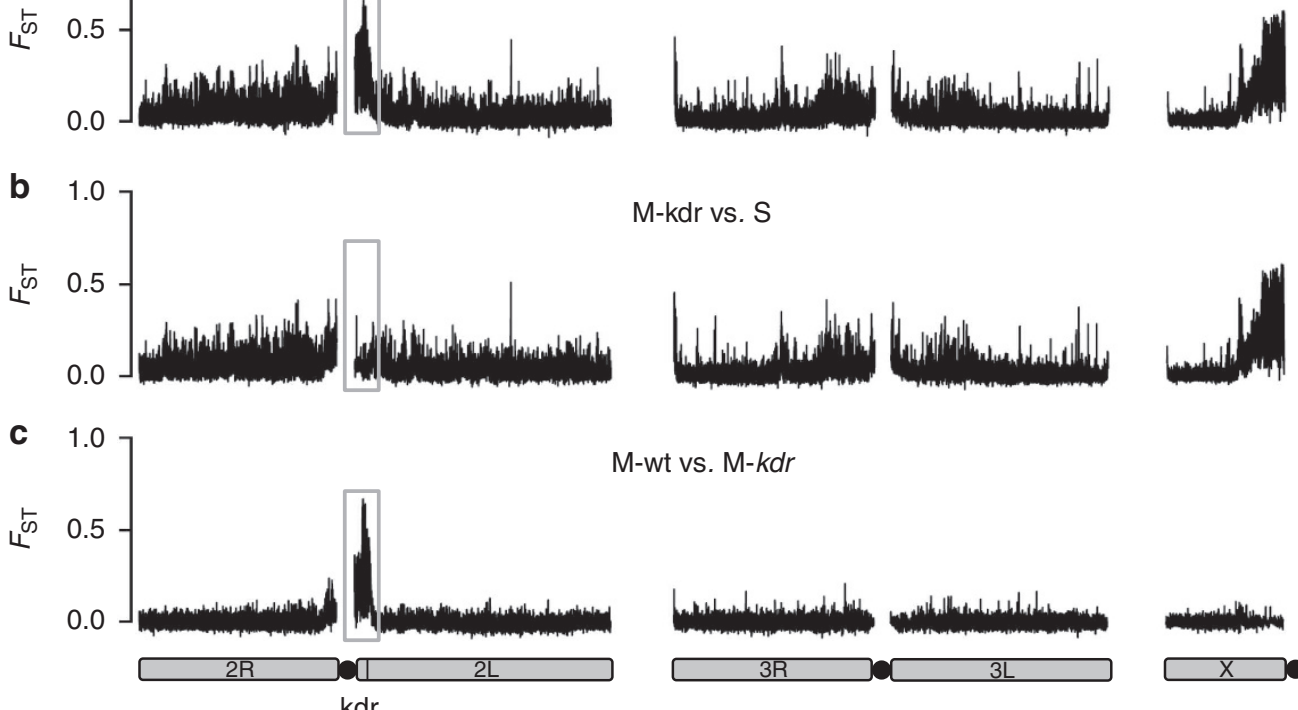

M-wt vs. M-kdr
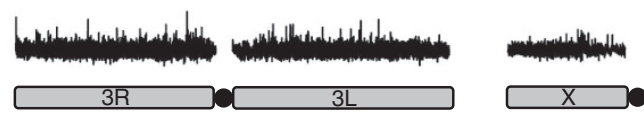

Figure 1 | Manhattan plots showing $\boldsymbol{F}_{\mathbf{S T}}$-based pairwise divergence between groupings of $\boldsymbol{A}$. gambiae. Plots are based on mean $F_{\mathrm{ST}}$ in 100-SNP stepping windows for (a) M-wt versus $\mathrm{S}$, (b) M-kdr versus $\mathrm{S}$, (c) M-wt versus M-kdr. Grey boxes highlight the $2 \mathrm{~L}$ genomic island region involved in introgression. Chromosomes are shown by solid grey bars and centromere positions by black circles. The position of the kdr (Vgsc-1014F) locus is shown on chromosome $\operatorname{arm} 2 \mathrm{~L}$. 
Malaria-transmitting mosquitoes are subjected to massive insecticidal pressure, which drives selection for rapid development of resistance ${ }^{32-34}$. Non-synonymous mutations in one of the two target sites for insecticides important in vector control, the voltage-gated sodium channel (Vgsc), are of particular significance. In A. gambiae the best known Vgsc mutation, L1014F, confers knockdown resistance $(k d r)$ to DDT and pyrethroids via a conformational alteration which reduces binding affinity of the insecticide ${ }^{35}$. In West Africa, Vgsc-1014F introgressed recently from $\mathrm{S}$ to $\mathrm{M}$ forms $\mathrm{s}^{30,36}$ and has subsequently increased dramatically in frequency in $\mathrm{M}^{33,37}$ consistent with strong anthropogenic selection ${ }^{33}$. The Vgsc is located within the large genomic island of divergence on chromosome arm 2L. Therefore, adaptive introgression and selection of Vgsc-1014F will result in reduced interform divergence, but the extent and impact of this genomic disruption is unknown. In A. gambiae from southern Ghana, where $\mathrm{M}$ and $\mathrm{S}$ are broadly sympatric, we show that the entire $2 \mathrm{~L}$ genomic island introgressed with apparently negligible impact on reproductive isolation during a period of rapid Vgsc-1014F increase, suggesting that it is neither critical to speciation nor maintained by strong divergent selection.

\section{Results}

Extent and impact of $k d r$ introgression. We sequenced the whole genomes of 15 wild-caught Ghanaian A. gambiae from three groups: S homozygous for the Vgsc-1014F kdr mutation; wild-type $\mathrm{M}$ that lack $k d r(\mathrm{M}-\mathrm{wt})$; and $\mathrm{M}$ homozygous for the $k d r$ allele that introgressed from $\mathrm{S}$ (M-kdr). Comparison of $\mathrm{M}$-wt and $\mathrm{S}$ form shows divergence across all chromosomes (Fig. 1a) concordant with previous low-density genome scans of Ghanaian $\mathrm{M}$ and $\mathrm{S}^{27,30}$ and high-density single-nucleotide polymorphisms (SNPs) genotyping of samples from Mali, Burkina Faso and Cameroon $^{28,29}$. However, the two large islands near the centromeres of $2 \mathrm{~L}$ and $\mathrm{X}$ identified originally ${ }^{12}$ are most prominent (Fig. 1a). Comparisons between the groups of samples show that over $3 \mathrm{Mb}$, representing approximately $1.5 \%$ of the genome and apparently encompassing the entire $2 \mathrm{~L}$ island of divergence, has introgressed between species. Consequently, divergence between $\mathrm{M}-k d r$ and $\mathrm{S}$ forms in this region of the genome has been eradicated (Fig. 1b), and in turn high, localized differentiation between M-kdr and M-wt created by introgression (Fig. 1c). Beyond the $2 \mathrm{~L}$ island the genomes of $\mathrm{M}-k d r$ and $\mathrm{M}-\mathrm{wt}$ are minimally differentiated (Fig. 1c), suggesting that either only the $2 \mathrm{~L}$ island region introgressed from $\mathrm{F}_{1}$ hybrids, or, perhaps more likely, that larger introgressed fragments have reduced in size through back-crossing and recombination within the $\mathrm{M}$ form.

We mapped the frequencies of $\mathrm{M}$ and $\mathrm{S}$ in larval collections from across southern Ghana (Fig. 2). Overall, $M$ and S were found at similar frequencies (55\%:45\%), and though relative frequencies varied considerably among locations, $\mathrm{M}$ and $\mathrm{S}$ cooccurred in 15 of the 18 collection sites. In southern Ghanaian M forms, $\mathrm{Vgsc}-1014 \mathrm{~F}$ is now present at consistently high frequency (mean \pm s.d. $=0.79 \pm 0.07$; range $=0.67-0.90)$, in marked contrast to when first detected in 2002 (Fig. 3a). This dramatic increase- to a frequency similar to that already present in $\mathrm{S}$ forms in 2002 (refs 38,39-is indicative of strong directional selection ${ }^{33}$. Despite the opportunity for hybridization afforded by widespread sympatry, frequencies of $\mathrm{M} / \mathrm{S}$ hybrids throughout the period of dramatic $k d r$ increase have remained low and stable (Fig. 3b). This suggests (i) minimal impact of introgression of the $2 \mathrm{~L}$ genomic island on reproductive isolation and (ii) that any divergent selection maintaining the island was much weaker than the directional selection driving the $k d r$ mutation to high frequency. We examined whether a relatively low frequency of

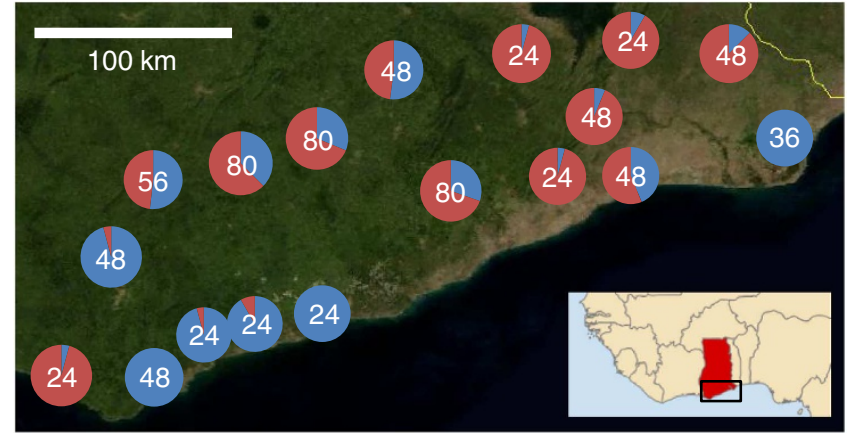

Figure 2 | Distribution of the $\mathbf{M}$ and $\mathbf{S}$ forms of $\boldsymbol{A}$. gambiae throughout southern Ghana. Pie charts show the relative frequency of $S$ form (shown in red) and $M$ form (in blue), from each site $(N=18)$ in collections made in 2011 (total $N=787$ ). The number collected at each site is shown in the centre of each pie chart.
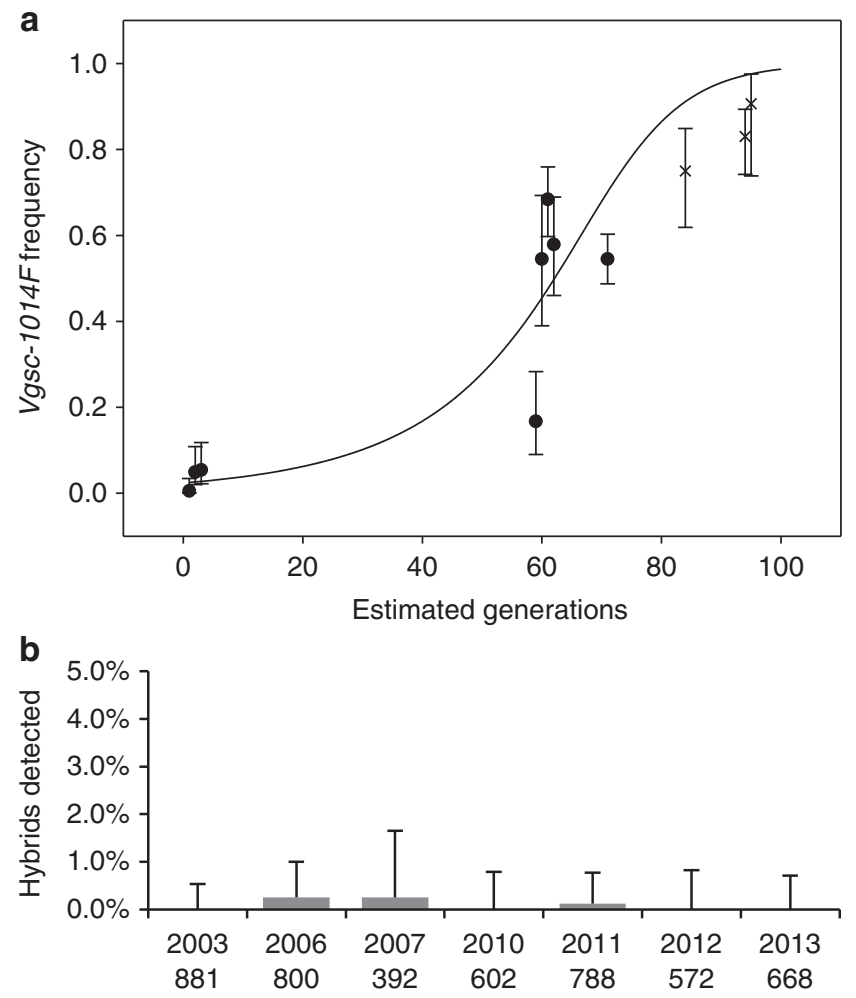

collection year (above), number of individuals (below)

Figure 3 | Spread of Vgsc-1014F kdr in M forms and M/S hybridization rates. (a) Increase in Vgsc-1014F frequency in M forms in Ghana, redrawn from the study by Lynd et al. ${ }^{33}$ with permission of Oxford University Press (points shown as filled circles) with additional data points (points shown as x). (b) Hybridization rates observed over a similar collection period with error bars showing binomial $95 \%$ upper confidence intervals. Sample size for each year is shown beneath the $X$ axis.

$\mathrm{S}$ forms in a collection site might limit opportunities for $k d r$ introgression into M. However, there was no difference in current $\mathrm{M}-k d r$ frequencies between sites where $\mathrm{S}$ forms were rare ( $\mathrm{S}$ frequency $0-0.09 ; k d r$ frequency $=0.78$ ) or those where they were common ( $\mathrm{S}$ frequency $0.48-0.96 ; k d r$ frequency $=0.82$; $t$-test; $t=0.82, P=0.41)$. Although relative frequencies of $\mathrm{M}$ and $S$ in sampling locations may have varied during the period of $k d r$ 

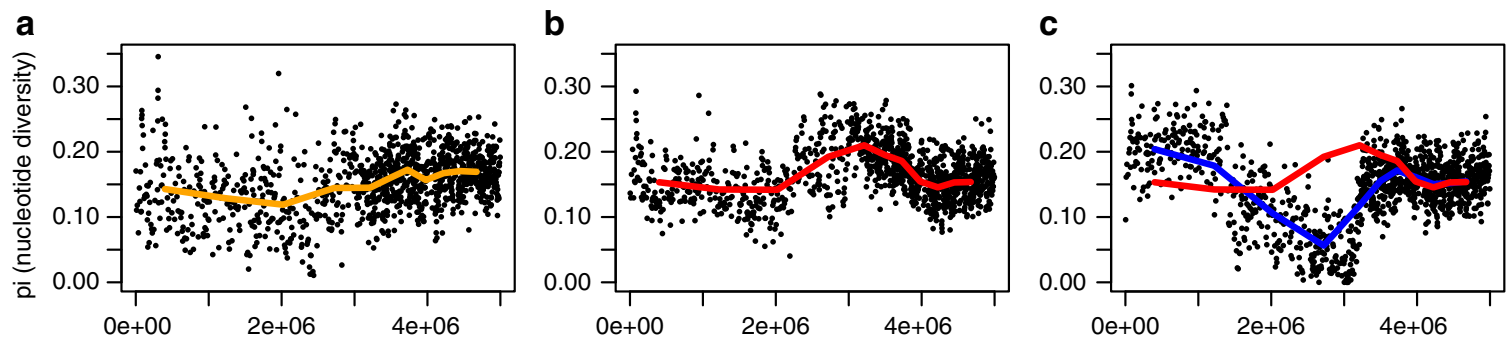

Distance along $2 \mathrm{~L}$ chromosome arm from centromere (nucleotides)

Figure 4 | Nucleotide diversity $(\pi)$ across the first $\mathbf{5} \mathbf{~ M b}$ of chromosome arm 2L, encompassing the genomic island region. For each sample group individual points represent mean $\pi$ in 100 bp stepping windows, whereas coloured lines are smoothed by using a $10 \mathrm{~kb}$ stepping window scale in (a) S form, (b) M-wt and (c) M-kdr, represented by the blue line, with the M-wt line (red) included for comparison.

increase, available evidence of no current association between relative $\mathrm{S}$ frequencies and $k d r$ frequency in the $\mathrm{M}$ form points to relatively infrequent introgression of $k d r$, rather than manifold introgression events. In the following sections we examine evidence that might explain how introgression of such a large, highly divergent fragment could spread so rapidly and without apparent impact on reproductive isolation via three hypotheses: (1) Only part of the $2 \mathrm{~L}$ island introgressed, without key loci involved in reproductive isolation; (2) The $2 \mathrm{~L}$ island is selectively unimportant as speciation is advanced and divergence is genome wide; (3) The divergence of the $2 \mathrm{~L}$ island results from processes reducing nucleotide diversity in low recombination regions rather than contemporary divergent selection.

Hypothesis 1. Visual inspection of $F_{\mathrm{ST}}$-based Manhattan plots (Fig. 1) suggest that the entire $2 \mathrm{~L}$ genomic island of divergence introgressed, but to examine this further we calculated mean pair wise nucleotide diversity $(\pi)$ from the centromere across the first $5 \mathrm{Mb}$ of the $2 \mathrm{~L}$ chromosome arm (numbering on $2 \mathrm{~L}$ starts at the centromere); a region exceeding the span of the genomic island. Neither $S$ nor M-wt exhibited any evidence of reduced $\pi$ (Fig. 4a,b), though the $\mathrm{S}$ form does experience localized lower $\pi$ relative to $\mathrm{M}$, possibly due to the effects of the historical sweep around $V g s c-1014 F$ in $S^{33}$. In contrast, and as expected in a region currently undergoing a selective sweep, the M- $k d r$ group shows a sharp drop in $\pi$ (Fig. 4 c). However, unlike $F_{\mathrm{ST}}$ (Fig. 1b,c), the signal from reduced nucleotide diversity does not span the entire $2 \mathrm{~L}$ island (Fig. 4c). To investigate this disparity in more detail, we first identified ancestry informative loci (that is, 'fixed' differences between the M-wt and S samples). Loci were then classified in each individual from the M-kdr sample as homozygous M-ancestry, homozygous S-ancestry or heterozygous (mixed ancestry) in the first $5 \mathrm{Mb}$ of the $2 \mathrm{~L}$ chromosome arm (Fig. 5). All M- $k d r$ samples showed M-ancestry from approximately $3.3-5 \mathrm{Mb}$ onwards, and in three of the five M- $k d r$ individuals, S-ancestry extended unbroken from approximately $3.3 \mathrm{Mb}$ back to the centromere. The other two $\mathrm{M}-k d r$ individuals showed near-perfect mixed ancestry in the first $1.4 \mathrm{Mb}$ of the chromosome arm, with an identical transition point to homozygous S-ancestry, indicating recombination at a single breakpoint within the $2 \mathrm{~L}$ island (Fig. 5). S-ancestry did not extend across the centromere into chromosome arm $2 \mathrm{R}$ in any $\mathrm{M}-k d r$ individual (Supplementary Fig. 1). The integrity of the $\mathrm{S}$ island in eight out of the ten M- $k d r$ chromosomes examined and the near 50:50 mixed ancestry in the other two from the centromere to the single shared breakpoint at $1.4 \mathrm{Mb}$, suggests that recombination is recent. Thus introgression most likely did result in transfer of the entire genomic island of divergence, which extends to $3.3 \mathrm{Mb}$, with recombination only just beginning to restore the $\mathrm{M}$ genomic background.

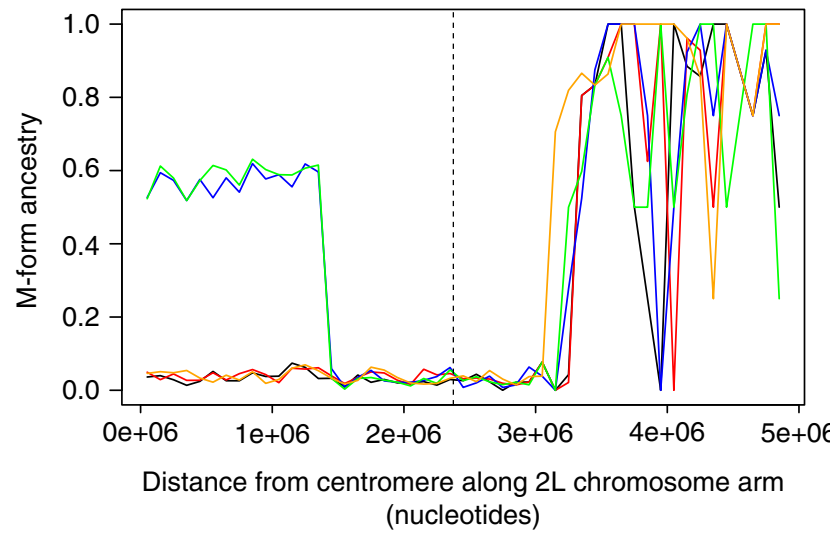

Figure 5 | Analysis of recombination within the introgressed 2 L genomic island. Lines show proportionate $M$ form ancestry for each individual in the $M-k d r$ group based on ancestry informative markers (fully diagnostic of $M$ and S). The black dashed line indicates the location of the voltage-gated sodium channel gene.

Hypothesis 2. Lack of impact of loss of the entire 2L genomic island might be because it merely represents the tip of a continuous distribution of divergence rather than a genomic island per se. To investigate this hypothesis we first examined the genomic distribution of $F_{\mathrm{ST}}$. In spite of the appearance of widespread, indeed potentially genome wide, differentiation between $\mathrm{M}$ and $\mathrm{S}$ in the Manhattan plots (Fig. 1a), inter-form differentiation is generally low, with a mean autosome-wide $F_{\text {ST }}$ $( \pm 95 \% \mathrm{CI})$ of only $0.032 \pm 0.0002$. Low genomic divergence, but high heterogeneity can be clearly seen from kernel density plots of the $F_{\mathrm{ST}}$ distributions for each chromosome arm (Fig. 6) and the associated skew and kurtosis statistics (Supplementary Table 1): all $\mathrm{M}$-wt versus $\mathrm{S}$-chromosome arm $F_{\mathrm{ST}}$ distributions are highly positively skewed and leptokurtic with long tails created by highly divergent SNPs (Fig. 6).

To facilitate precise localization of areas of marked divergence (putative genomic islands) we utilized the ancestry informative loci, this time across the whole genome $(0.24 \%$ of all $13,924,420$ SNPs). From the proportion of fixed differences within $50 \mathrm{~kb}$ windows (fixed difference, $d_{\mathrm{f}}$ ) we defined non-contiguous windows significantly enriched for ancestry informative loci as distinct putative genomic islands of divergence. Plots of $d_{\mathrm{f}}$ suggest the presence of genomic islands (Fig. 7), albeit highly variable in size and number across chromosome arms (Table 1, Supplementary Data 1). Over $80 \%$ of the putative islands are small, comprising of three or fewer adjacent significant $50 \mathrm{~kb}$ windows (Table 1 ), whereas three were very large, the $2 \mathrm{~L}$ island 
$(3.3 \mathrm{Mb})$ and the two adjacent pericentromeric $\mathrm{X}$ islands (1.45 and $4.9 \mathrm{Mb}$ ), which were likely merged in earlier low resolution analyses $^{12,31}$. Maximum and mean $d_{\mathrm{f}}$ were very strongly correlated with one another (Supplementary Table 2) and with island size (Supplementary Fig. 2a,b), that is, islands with higher $d_{\mathrm{f}}$ also tended to cover larger areas. Among islands both mean and maximum $d_{\mathrm{f}}$ were significantly positively correlated with SNP frequency (Supplementary Table S2), and though island size was not, it was notable that the largest islands had relatively few SNPs (Supplementary Fig. 2c) and also relatively few genes (Supplementary Fig. 2d). This contrast highlights the different patterns of polymorphism between smaller and very large islands, with the former exhibiting increasing SNP frequency with size, a relationship which breaks down for the largest islands. Our results suggest that genomic divergence between $M$ and $S$ is both highly heterogeneous and largely restricted to islands. Moreover, the very large islands on $2 \mathrm{~L}$ and $\mathrm{X}$ remain almost as prominent as originally suggested in early low-resolution scanning ${ }^{12}$ and contain almost $45 \%$ of all significantly differentiated windows. In summary, though islands appear both far more numerous and thus cover more of the genome than originally thought ${ }^{12}$, divergence appears too heterogeneous in both island size and distribution to be considered as genome wide ${ }^{40,41}$; therefore hypothesis 2 is not supported.

Hypothesis 3. Our data suggest that genomic divergence between $\mathrm{M}$ and $\mathrm{S}$ is appropriately described by an island model, albeit one involving many islands. Detailed recombination rate data are currently unavailable for the A. gambiae genome, but the location of the $2 \mathrm{~L}$ island and the largest islands on the $\mathrm{X}$ chromosome near centromeres suggests that they are likely to experience

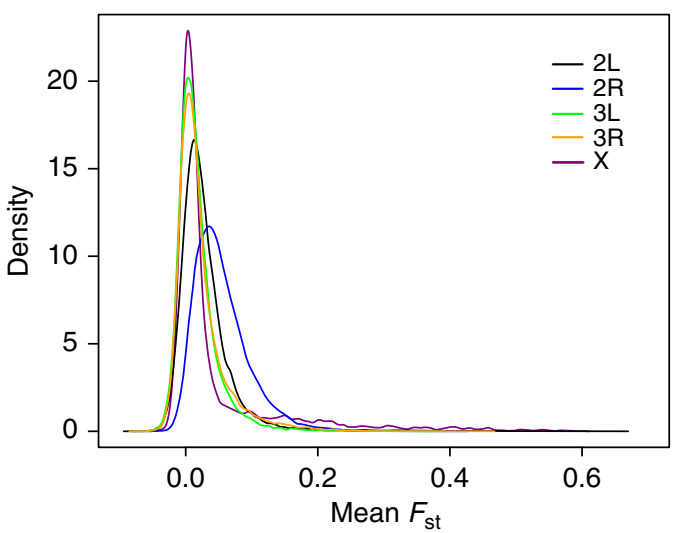

Figure 6 | Kernel density plots of $\boldsymbol{F}_{\mathrm{ST}}$ for $\boldsymbol{M}$-wt versus $\mathbf{S}$ for each chromosome arm. Distributions of mean $F_{\mathrm{ST}}$, calculated over 100-SNP stepping windows for all chromosome arms. reduced recombination $6,7,10,42,43$, which is consistent with their relatively low gene and SNP densities. $F_{\mathrm{ST}}$ is inversely related to genetic diversity (estimated by number of segregating sites per window-Supplementary Fig. 3), and strong differentiation could reflect the actions of forces, other than contemporary divergent selection, that reduce diversity which is then very slow to recover in low recombination regions ${ }^{7,11}$. Consequently, we examined additional metrics for evidence of selection operating on islands, which might provide a means of partitioning historical signals of reduced diversity from recent divergent selection ${ }^{7,9,11}$. We first calculated Dxy ${ }^{44}$, a measure of absolute divergence of all nucleotide positions in a sequence, for $50 \mathrm{~kb}$ windows. Nevertheless, caution is required in application of Dxy, which is prone to high variance with smaller sample size, and is known to exhibit high stochastic variance among $\mathrm{SNPs}^{45}$, both of which might affect genome scan analyses. Consistent with, for example, the effects of background selection in low recombination regions $^{7,9}$, Dxy was depressed near centromeres (Supplementary Fig. 4) and peaks were not coincident with the islands identified using $d_{\mathrm{f}}$ (only one out of the 436 windows which comprise the islands exceeded a 0.99 th percentile of Dxy). Such observations would appear to support hypothesis 3, that the islands could reflect historical rather than contemporary selective events ${ }^{7}$. However, Dxy was highly positively correlated with SNP frequency of islands $(\rho=0.89, P<0.001)$ and also with its standard deviation within windows $(\rho=0.98, P<<0.001)$. Indeed, the relationship between the mean and standard deviation of Dxy is higher for islands generally, and the three very large islands (on $\mathrm{X}$ and $2 \mathrm{~L}$ ) show especially extreme relative standard deviation (Fig. 8). In other words, the islands identified using $d_{\mathrm{f}}$ did contain large values of Dxy, but many monomorphic sites (that is, a low SNP frequency) inevitably reduce values across a window, leading to exceptional variance for a given Dxy value.

Table 1 | Size distribution of islands divergent between $M$ and $\mathbf{S}$.

\begin{tabular}{lrrrrrrc} 
Size class (bp) & $\mathbf{2 L}$ & $\mathbf{2 R}$ & $\mathbf{3 L}$ & $\mathbf{3 R}$ & $\mathbf{X}$ & Total & Cumulative \% \\
\hline 50,000 & 10 & 29 & 7 & 16 & 2 & 64 & 55 \\
100,000 & 1 & 10 & 1 & 3 & 3 & 18 & 70 \\
150,000 & 2 & 6 & 1 & 4 & 0 & 13 & 81 \\
200,000 & 0 & 2 & 0 & 2 & 0 & 4 & 85 \\
250,000 & 0 & 4 & 0 & 0 & 0 & 4 & 88 \\
300,000 & 0 & 3 & 0 & 2 & 0 & 5 & 92 \\
350,000 & 0 & 3 & 0 & 0 & 0 & 3 & 95 \\
400,000 & 0 & 0 & 0 & 1 & 0 & 1 & 96 \\
450,000 & 0 & 0 & 0 & 0 & 0 & 0 & 96 \\
$500,000+$ & 0 & 2 & 0 & 0 & 0 & 2 & 97 \\
$1,000,000+$ & 1 & 0 & 0 & 0 & 2 & 3 & 100
\end{tabular}

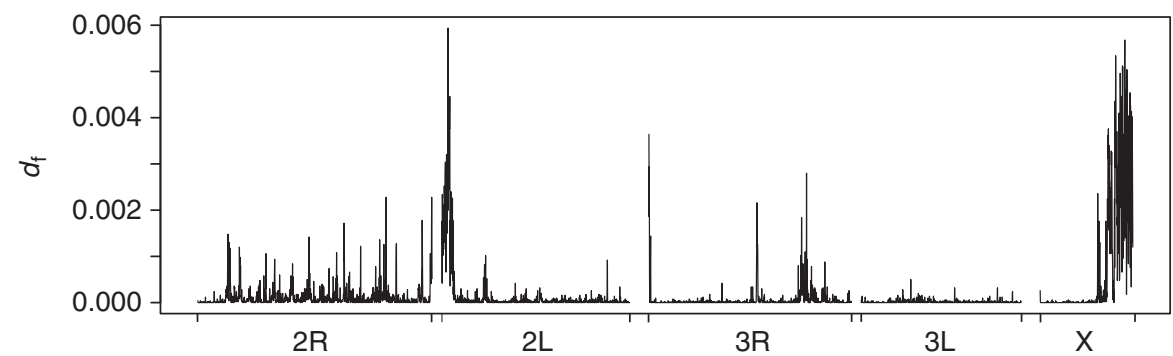

Figure 7 | Genomic landscape of divergence between $\mathbf{M}$ and S. The density of fixed differences (SNPs) between M-wt and S ( $d_{\mathrm{f}}$ ) in $50 \mathrm{~kb}$ stepping windows. Chromosomes and centromere position are shown by grey bars and black circles respectively; the position of the $k d r$ Vgsc-1014F locus is shown. 
This will render Dxy extremely sensitive to the size of particular windows, with potential for ambiguous interpretation. Therefore, if covariates affecting Dxy are considered it could not usefully provide discrimination of competing hypotheses for our dataset. Moreover, we note a high correlation $(r=-0.5)$ between sequence depth and Dxy, suggesting sensitivity to sequencing error.

Second we calculated Tajima's $\mathrm{D}^{46}$, again for $50 \mathrm{~kb}$ windows; extreme values of $\mathrm{D}$ result from an imbalance between pairwise nucleotide diversity and the number of segregating sites, with negative values potentially indicating directional selection and high values, balancing selection. In contrast to Dxy, a low correlation between sequence depth and Tajima's D was found $(r=0.19)$.

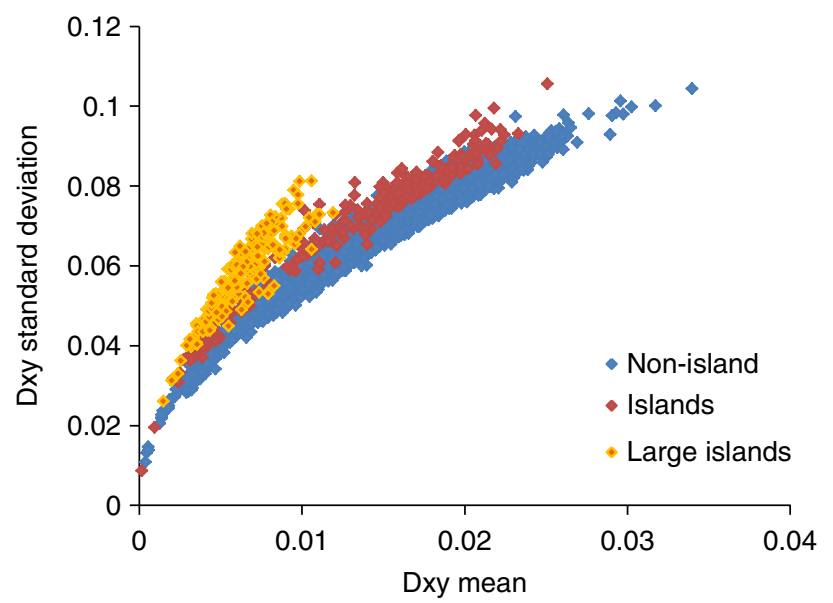

Figure 8 | Scatterplot of absolute divergence, Dxy, plotted against its standard deviation. Points are means for every $50 \mathrm{~kb}$ window in the genome, with colours denoting windows from genomic islands of divergence (red), those from the three largest islands of divergence (those over $1 \mathrm{Mb}$ in size), two on the $\mathrm{X}$ chromosome and one on $2 \mathrm{~L}$ (yellow) and the windows from the rest of the genome (blue).
There was no signal of directional selection around the $2 \mathrm{~L}$ island in any group (Fig. 9), with $\mathrm{S}$ forms actually exhibiting the highest positive values of Tajima's $\mathrm{D}$ within the $2 \mathrm{~L}$ island, indicative of balancing selection (Supplementary Fig. 5). This counterintuitive result seems unlikely to reflect balancing selection, but is concordant with theoretical expectations for a positive Tajima's $\mathrm{D}$ signal ${ }^{47}$ arising from a secondary selective sweep of the region, which overlays an earlier sweep likely driven by $\mathrm{Vgsc}-1014 \mathrm{~F}$. The recently discovered resistance allele $\operatorname{Vgsc}-1575 \mathrm{Y}^{34}$ could provide a plausible candidate, as all the $\mathrm{S}$-form individuals sequenced were $\mathrm{N} 1575 \mathrm{Y}$ heterozygotes, although at around $3 \mathrm{Mb}$ on 2L, the peak of Tajima's D is offset from the Vgsc.

Highly negative values of Tajima's D were almost entirely absent from the autosomes of $\mathrm{M}$ and $\mathrm{S}$; though peaks were found throughout both of the two centromere-proximal $\mathrm{X}$ chromosome islands in $\mathrm{M}$ forms (Fig. 9). In S, there was a notable clustering of negative Tajima's D peaks centred around $18.5 \mathrm{Mb}$, with extreme values (exceeding a two-tailed 99\% threshold) just extending into the beginning of the largest $X$ island (Fig. 9) and thus clearly offset from peak interform divergence on $\mathrm{X}$ (Figs 1 and 7). Coincident outlying negative values were also found in the smaller island region within both $\mathrm{M}$ groups. Gene annotation term enrichment analysis identified a significant overrepresentation of genes linked with chitin synthesis genes in this region (Supplementary Table 3). The negative Tajima's D signals throughout the largest $\mathrm{X}$ islands in both $\mathrm{M}$ groups, but not in $\mathrm{S}$, suggest selection potentially acting within $\mathrm{M}$ forms rather than divergent selection acting on both $\mathrm{M}$ and $\mathrm{S}$, as found in the smaller X island.

The relationship between Dxy and its variance suggests unreliability because of extreme dependence on window size and a concerning potential for sensitivity to sequencing error. Tajima's D suggested some concordance of divergent regions with selection, though the likely conflation of signals within the $2 \mathrm{~L}$ island region highlights that problems can occur with interpretation. However, Tajima's D yielded some signals of selection for each of the large $\mathrm{X}$ islands. In particular, the secondary island

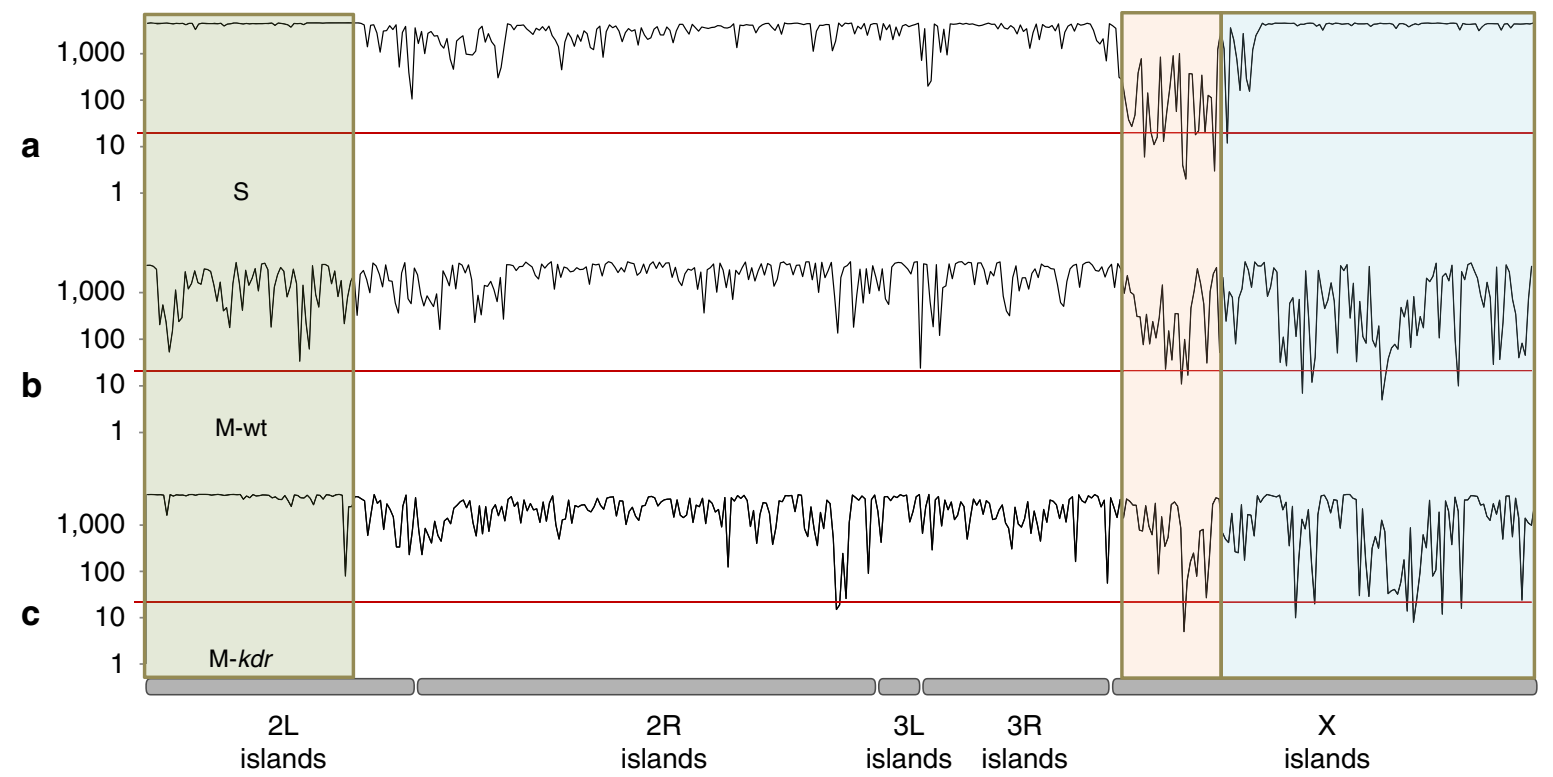

Figure 9 | Evidence of directional selection from Tajima's D across all genomic islands in each group. Plots show ranks of Tajima's D negative values (lower = more negative) for the 438 significant windows comprising islands, arrayed in order of physical position on each chromosome arm for the three sample groups: (a) S form, (b) M-wt and (c) M-kdr. Ranks are initially calculated across all 4,612 windows within each group, but only island window are shown. The red line shows the two-tailed lower 99 th percentile rank used as a threshold for extreme values. Windows within the major $2 \mathrm{~L}$ island and in the pair of very large islands of divergence on the $\mathrm{X}$ chromosome are highlighted in shaded boxes. 
region on $\mathrm{X}$, which lies outside of the pericentromeric heterochromatin region of extreme low recombination ${ }^{43}$, is both significantly divergent between $M$ and $S$, and shows evidence of contemporary directional selection operating within $\mathrm{M}$ and $\mathrm{S}$, supporting a novel hypothesis of involvement in ongoing divergence. In summary, though hypothesis 3 is difficult to disprove conclusively, Tajima's D provided evidence of ongoing selection on the $\mathrm{X}$ islands (if not for the $2 \mathrm{~L}$ island), and highlights the utility of combining multiple metrics in candidate region discovery.

\section{Discussion}

In this study we investigated a case of introgression between the most recently diverged species within the A. gambiae complex. The adaptive nature of the $V g s c 1014 F$ mutation is clearly evident from its significant association with insecticide resistance ${ }^{34}$ and its dramatic rate of increase in A. gambiae $\mathrm{M}$ forms. Introgression of $\mathrm{Vgsc1014F}$ from $\mathrm{S}$ to $\mathrm{M}$ forms has also been documented in Benin $^{36}$, Cameroon ${ }^{48}$ and Burkina Faso ${ }^{37}$, with a similarly rapid increase in frequency observed in the latter. Moreover, though not explicitly considered by the authors, temporal variation in numbers of hybrids and back-crosses detected in a longitudinal study of a single village in $\mathrm{Mali}^{26}$ might be linked to selection for introgression of $\mathrm{Vgsc}-1014 \mathrm{~F}$ into $\mathrm{M}$ forms, rather than relaxed selection against hybrids and back-crosses linked to other unknown environmental variations ${ }^{26}$. The present data are unique in demonstrating the genomic extent of introgression. However, studies of introgression from Mali and Cameroon, albeit based on only one or two SNPs in the 2L genomic island region outside of the $\operatorname{Vgsc}^{26,27}$, and also the variety of locations from which $k d r$ introgression has been recorded, suggest that our results are very unlikely to be restricted to southern Ghana.

Given the location of the $V g s c$ gene in the 2L pericentromeric region, which is thought to exhibit low recombination ${ }^{29,49}$, we hypothesized that a relatively extensive area might be affected by the selective sweep. In fact, the impacted area proved to be huge, exceeding $3 \mathrm{Mb}$ ( $1.5 \%$ of the genome), and spanned the entirety of one of the two most prominent genomic islands of divergence between $\mathrm{M}$ and $\mathrm{S}$. Coupled with hybridization data collected during the period of $V g s c 1014 F$ increase in $\mathrm{M}$ forms, this provided a natural test of whether the loss of a major genomic island of divergence reduces the reproductive isolation of $\mathrm{M}$ and $\mathrm{S}$, as might be expected if the island contained genes critical to the speciation process; that is, a 'speciation island'12. Our results do not support the designation of the $2 \mathrm{~L}$ genomic island of divergence as a speciation island. $\mathrm{M}$ and $\mathrm{S}$ forms are extensively sympatric across southern Ghana, presenting widespread opportunity for hybridization. Yet hybridization rates appear stable throughout the period of rapid increase of introgressed $V g s c 1014 F$ to high frequency in $\mathrm{M}$ form populations across southern Ghana. It would appear that transfer of the entire island has had no discernible impact on reproductive isolation, allowing effective co-option of the adaptive $V g s c-1014 F$ mutation into the $\mathrm{M}$ genomic background via adaptive introgression. The large pericentromeric speciation islands on separate chromosomes (X, $2 \mathrm{~L}$ and $3 \mathrm{~L}$ ) are usually in strong linkage disequilibrium, which could imply epistatic selection ${ }^{10,31}$. If this were the case, it seems unlikely that the genome of $\mathrm{M}$ forms could tolerate such massive disruption without a major loss of fitness. By contrast, our results suggest that any $\mathrm{M}$ form fitness cost is overcome by the increase in fitness from gaining the $V g s c-1014 F$ mutation. It would seem therefore, that any selective importance of the $2 \mathrm{~L}$ island of divergence does not arise from its impact on reproductive isolation, and that it is not currently involved in speciation. Though some past involvement in divergence cannot be ruled out, our results highlight that large areas of interform divergence, however eye-catching, are not necessarily under selective forces proportional to their size.

Reduced haplotypic diversity in the $V g s c$ of S forms is evidence for recent selection ${ }^{33}$, which, before introgression of the $V g s c$ $1014 \mathrm{~F}$ mutation and increase to high frequency in $\mathrm{M}$ forms, would have resulted in increased divergence. Although interpretation of the strong Tajima's D signal of selection on $2 \mathrm{~L}$ was ambiguous, and given low recombination in the $2 \mathrm{~L}$ island, it is possible that a portion extending some way beyond the Vgsc might have been subjected to the sweep of $V g s c-1014 F$. This poses the question of whether $\mathrm{M}$ and $\mathrm{S}$ divergence on $2 \mathrm{~L}$ is simply a result of selection operating within S forms. Selection on $\mathrm{Vgsc}$ $1014 F$ can be discounted as a general explanation because divergence in this region was first documented from comparison of $\mathrm{M}$ and $\mathrm{S}$, which both lacked the $V g s c-1014 \mathrm{~F}$ mutations ${ }^{12}$. Selection within $\mathrm{S}$ alone is also not supported by comparative patterns of nucleotide diversity in the island region, levels of which are broadly similar in $\mathrm{M}$-wt and $\mathrm{S}$ across the island region despite the historical selective sweeps in $S^{33,34}$ (Fig. 4a,b). Apart from recent selection on $V g s c-1014 F$, is the $2 \mathrm{~L}$ island under any contemporary directional selection at all or is its size an artefact of background selection? Unfortunately, the additional metrics we applied (Dxy and Tajima's D) did not allow separation of these hypotheses but selection on $V g s c-1014 F$ provides some additional insight. Given the very rapid increase in $V g s c-1014 \mathrm{~F}$ frequency in $M$ forms following introgression, any negative fitness consequences resulting from loss of alleles under selection within the $2 \mathrm{~L}$ island must have been outweighed by insecticidal selection on $V g s c-1014 F$, for which we have estimated a selection coefficient of $s=0.16$ (ref. 33). From the size of the introgressed fragment, it is now apparent that this represents a net estimate for the $3.3 \mathrm{Mb} 2 \mathrm{~L}$ island of divergence, rather than $\mathrm{Vgsc}-1014 \mathrm{~F}$ alone. Thus, either selection on $V g s c-1014 F$ is much stronger than initially estimated or the total selection acting on all variants within the $2 \mathrm{~L}$ island of divergence is weak.

If selection on such a large island appears weak, it is natural to question the importance of the other, often small, islands throughout the genome and whether reduced recombination plays a key role in their formation ${ }^{8}$. SNP frequency data do not support the latter for many of the smaller islands, which, in contrast to the very large islands, often showed quite high densities of segregating sites, at odds with the expectation for a low recombination region. Nevertheless, differentiation of so many islands seems puzzling unless they are by-products of genome-wide divergence, which does not appear to fit their heterogeneity in size and distribution. To account for the genomically widespread differences between $\mathrm{M}$ and $\mathrm{S}$, when there is clear evidence for recent gene flow, Reidenbach et al. ${ }^{28}$ proposed an 'extrinsic' environmental hypothesis. In this scenario, hybridization occurs in infrequent bursts during unusual environmental conditions, with strong selection against introgressed individuals when typical conditions return $^{28}$. Recent data from a time series study in a Malian village appear consistent with this hypothesis ${ }^{26}$, though as noted above $V g s c-1014 \mathrm{~F}$ introgression might also be involved. An alternative, and not mutually exclusive 'intrinsic' hypothesis, is that the nature of the genomic landscape of divergence provides permissiveness to gene flow. Selection dispersed across many islands is likely to be weak for the majority of loci, potentially enabling resilience to temporary loss of (weakly selected) islands until they can be restored by back-crossing. Searching for 'individual speciation genes' in such a landscape will thus be difficult because of low selection coefficients and frequent lack of correspondence between significant divergence and functionality. 
Our results provide a natural 'loss of function' test of the $2 \mathrm{~L}$ island, which bears many similarities in terms of likely recombination profile, polymorphism and divergence to the only other exceptionally large islands, located on the $\mathrm{X}$ chromosome. We think it is unwise to extrapolate from these commonalities a similar lack of importance for the $\mathrm{X}$ islands in speciation between $\mathrm{M}$ and S. X (or Z) chromosomes evolve relatively quickly owing to reduced effective population size and are known to be critically involved in the development of reproductive isolating mechanisms between many species ${ }^{50}$, including other, less closely related members of the A. gambiae species complex ${ }^{51,52}$. Moreover, and although proof of a speciation island must come from demonstrable function, Tajima's $\mathrm{D}, F_{\mathrm{ST}}$ and $d_{\mathrm{f}}$ all provide signals consistent with selection acting around $17-19 \mathrm{Mb}$ on the $\mathrm{X}$ chromosome in both $\mathrm{M}$ and $\mathrm{S}$, and perhaps further towards the centromere in $\mathrm{M}$ forms. The signal of selection found in both $\mathrm{M}$ and $\mathrm{S}$ was primarily focused on the smaller of the two major $\mathrm{X}$ islands, the physical distance of which from the centromere may make it more likely that selection rather than just low recombination preserves its large size. Interestingly, while the largest island on $\mathrm{X}$ is always present when comparing $\mathrm{M}$ and $\mathrm{S}$, this secondary $\mathrm{X}$ island area is absent from locales such as Guinea-Bissau, The Gambia and Senegal exhibiting exceptionally high hybridization rates ${ }^{27,53}$. Follow-up studies on the role of this genomic region are warranted.

A multi-locus, resilient genomic architecture of divergence presents an interesting paradox for speciation theory. Typically, the presence of substantial gene flow has been viewed as a signal of early-stage incipient speciation $^{22}$, some way from the degree of reproductive isolation at which organisms might be recognized as 'good species'. However, it is becoming recognized now that gene flow between closely related 'good species' is extremely widespread $^{40,54}$. If selection is spread across numerous loci this may effectively provide intrinsic redundancy, and interspecific gene flow may actually be a long-lasting, stable state. A genomic landscape of generally weak but highly heterogeneous differentiation, though perhaps far from a highly differentiated 'end point ${ }^{40}$ expected for species, may be an important stage in genomic divergence, which can allow both adaptive introgression and protection of reproductive isolation. The molecular forms have recently been reclassified as A. gambiae s.s. and Anopheles coluzzii ${ }^{15}$, based primarily on evidence of reproductive isolation from relatively widespread genomic differentiation ${ }^{28,29,55}$ and partial ecological niche partitioning ${ }^{56,57}$. While we do not interpret our data as revealing truly genome-wide divergence, under either the 'extrinsic' or 'intrinsic' hypotheses outlined above, our results support this reclassification of $\mathrm{M}$ and $\mathrm{S}$ forms as species.

\section{Methods}

Collections. Adult female mosquitoes used subsequently for whole-genome sequencing were collected by aspiration from southern Ghana during the summer of 2007. Six locations (Supplementary Table 4) were sampled to yield five A. gambiae $\mathrm{S}$ form (individuals homozygous for the $\mathrm{Vgsc}-1014 \mathrm{~F}$ mutation) and ten A. gambiae M form (five individuals homozygous for the wild-type 1014L and five homozygous for the introgressed $V g s c-1014 F$ mutation). $1014 \mathrm{~F}$ is close to fixation in A. gambiae $\mathrm{S}$ form populations in this region, so no homozygote $1014 \mathrm{~L}$ individuals were available. Multiple collection locations were necessary due to a sequencing protocol that required a high yield of extracted DNA. Before extraction all samples were stored dry over silica. Data for M/S hybrid frequencies in southern Ghana were collated from collections we have described elsewhere ${ }^{30,38,39,58,59}$.

DNA extraction and sequencing. After morphological identification as A. gambiae s.l., DNA was extracted from dried whole bodies using the DNeasy extraction kit (Qiagen). Species (within A. gambiae s.l.) were identified using a standard PCR diagnostic assay ${ }^{60}$, with subsequent identification of molecular forms using the SINE diagnostic method ${ }^{61}$. As Ghanaian sampling was concerned with $k d r$ introgression, these 15 individuals were also genotyped for the presence of the voltage-gated sodium channel mutation $V g s c-1014 F$ using a TaqMan assay ${ }^{62}$. DNA quantification was carried out using Quant-iT PicoGreen dsDNA fluorimetric assays (Invitrogen), taking the mean concentration from two technical replicates. Sample libraries were cloned with $200-300$ bp inserts, and 76 bp pairedend sequencing was conducted using an Illumina High Seq 2000 by the Wellcome Trust Sanger Institute. Reads were aligned to the AgamP3 reference genome ${ }^{63}$ using BWA ${ }^{64} ; 82-90 \%$ of read pairs per sample successfully aligned to the reference sequence, giving a median read depth per sample of 7-20x. Variant calling was conducted using SAMtools mpileup and BCFtools to produce a raw vcf file, which was filtered using vcfutils.pl varFilter-D100 (ref. 65). Non-biallelic SNP loci were removed using VCFtools ${ }^{66}$. Sequenced individuals were re-checked for read depth, particularly in regions of interest, and molecular form was validated in the genome sequence data via presence/absence of the SINE insertion ${ }^{61}$ using LookSeq ${ }^{67}$.

Statistical analyses. Genomic divergence between mosquito sample groups and pairwise nucleotide diversity within groups were calculated for every SNP using VCFtools version 0.1.9.0 (ref. 66) (commands) via the Weir and Cockerham estimator of $F_{\mathrm{ST}}$ (- -weir-pop-fst) and $\pi$ (- -site-pi), respectively. 'Fixed' differences in $F_{\mathrm{ST}}$ between the $\mathrm{S}$ and $\mathrm{M}$-wild-type sample groups were identified and used (1) as ancestry informative markers to study localized recombination in the M-kdr group, and (2) to estimate the proportion of $d_{\mathrm{f}}$ (ref. 68) within non-overlapping $50 \mathrm{~kb}$ windows. This represents an arbitrary size that simply represents a balance between resolution and minimizing impacts of any SNP calling errors, and is not tailored to any specific detailed recombination map as this is unavailable for $A$. gambiae. Plots of $F_{\mathrm{ST}}, d_{\mathrm{f}}$ and $\pi$ against chromosomal position were produced from means of windows with the statistical software package $\mathrm{R}^{69}$ and custom Perl and $\mathrm{R}$ scripts. A 100-SNP stepping window size was chosen to visualize $F_{\mathrm{ST}}$ because this has been shown to produce accurate estimates of Weir and Cockerham's $F_{\mathrm{ST}}$ from low sample sizes ${ }^{70}$. To identify putative genomic islands of divergence, we tested for exceptional values of $d_{\mathrm{f}}$ by simulating 100,000 Poisson distributions based on the actual number of windows and ancestry informative markers and applying a window-specific threshold scaled according to the SNP frequency within the window. As a conservative threshold for identification of clustering within a window we applied a Bonferroni-corrected upper percentile limit for $d_{\mathrm{f}}$ from simulations: only observed $d_{\mathrm{f}}$ values exceeding this were considered significant. Adjacent significant windows were considered part of the same island; however, we considered islands as continuous if a nonsignificant window intervened between significant windows, but this exceeded the upper 0.8 percentile limit of simulated $d_{\mathrm{f}}$. Kernel plots and associated skewness and kurtosis statistics were used to study the density distributions of $F_{\mathrm{ST}}$ values for each SNP on each chromosome and were calculated and plotted using custom Perl scripts and R (ref. 69). Additional metrics to study variation in the pairwise polymorphic site-frequency spectrum between individuals within a sample and absolute divergence between samples were calculated as Tajima's $\mathrm{D}^{46}$ and $\mathrm{Dxy}^{45}$, respectively, using custom Perl scripts and $\mathrm{R}$ (ref. 69). Sequence data was 'haploidized' for estimation of these parameters (following Ellegren et al. ${ }^{68}$ ) by randomly assigning alleles at heterozygous positions. Spearman correlation coefficients between descriptive statistics (none of which were normally distributed) for islands were calculated using SPSS v20 (IBM, Armonk, NY)

\section{References}

1. Hedrick, P. W. Adaptive introgression in animals: examples and comparison to new mutation and standing variation as sources of adaptive variation. Mol. Ecol. 22, 4606-4618 (2013).

2. Song, Y. et al. Adaptive introgression of anticoagulant rodent poison resistance by hybridization between old world mice. Curr. Biol. 21, 1296-1301 (2011)

3. Pardo-Diaz, C. et al. Adaptive introgression across species boundaries in Heliconius butterflies. PLoS Genet. 8, e1002752 (2012).

4. Kronforst, M. R. et al. Hybridization reveals the evolving genomic architecture of speciation. Cell Rep. 5, 666-677 (2013).

5. Hansen, T. F. Why epistasis is important for selection and adaptation. Evolution 67, 3501-3511 (2013).

6. Carneiro, M., Ferrand, N. \& Nachman, M. W. Recombination and speciation: loci near centromeres are more differentiated than loci near telomeres between subspecies of the European rabbit (Oryctolagus cuniculus). Genetics 181, 593-606 (2008).

7. Noor, M. A. F. \& Bennett, S. M. Islands of speciation or mirages in the desert? Examining the role of restricted recombination in maintaining species. Heredity 103, 439-444 (2009).

8. Renaut, S. et al. Genomic islands of divergence are not affected by geography of speciation in sunflowers. Nat. Commun. 4, 1827 (2013).

9. Charlesworth, B., Nordborg, M. \& Charlesworth, D. The effects of local selection, balanced polymorphism and background selection on equilibrium patterns of genetic diversity in subdivided populations. Genet. Res. 70, 155-174 (1997).

10. Turner, T. L. \& Hahn, M. W. Genomic islands of speciation or genomic islands and speciation? Mol. Ecol. 19, 848-850 (2010).

11. Cutter, A. D. \& Payseur, B. A. Genomic signatures of selection at linked sites: unifying the disparity among species. Nat. Rev. Genet. 14, 262-274 (2013).

12. Turner, T. L., Hahn, M. W. \& Nuzhdin, S. V. Genomic islands of speciation in Anopheles gambiae. PLoS Biol. 3, e285 (2005). 
13. Turner, T. L. \& Hahn, M. W. Locus- and population-specific selection and differentiation between incipient species of Anopheles gambiae. Mol. Biol. Evol. 24, 2132-2138 (2007).

14. della Torre, A. et al. Molecular evidence of incipient speciation within Anopheles gambiae s.s. in West Africa. Insect Mol. Biol. 10, 9-18 (2001).

15. Coetzee, M. et al. Anopheles coluzzii and Anopheles amharicus, new members of the Anopheles gambiae complex. Zootaxa 3619, 246-274 (2013).

16. Lehmann, T. \& Diabate, A. The molecular forms of Anopheles gambiae: a phenotypic perspective. Infect. Genet. Evol 8, 737-746 (2008).

17. Diabaté, A. et al. Spatial swarm segregation and reproductive isolation between the molecular forms of Anopheles gambiae. Proc. Biol. Sci. B 276, 4215-4222 (2009).

18. Dabiré, K. R. et al. Assortative mating in mixed swarms of the mosquito Anopheles gambiae s.s. $\mathrm{M}$ and S molecular forms, in Burkina Faso, West Africa. Med. Vet. Entomol. 27, 298-312 (2013)

19. Diabaté, A., Dabire, R. K. \& Millogo, N. Evaluating the effect of postmating isolation between molecular forms of Anopheles gambiae (Diptera: Culicidae). J. Med. Entomol. 44, 60-64 (2007).

20. Della Torre, A., Tu, Z. \& Petrarca, V. On the distribution and genetic differentiation of Anopheles gambiae s.s. molecular forms. Insect Biochem. Mol. Biol. 35, 755-769 (2005)

21. Wu, C.-I. The genic view of the process of speciation. J. Evol. Biol. 14, 851-865 (2001).

22. Wu, C.-I. \& Ting, C.-T. Genes and speciation. Nat. Rev. Genet. 5, 114-122 (2004).

23. Smadja, C., Galindo, J. \& Butlin, R. Hitching a lift on the road to speciation. Mol. Ecol. 17, 4177-4180 (2008)

24. Via, S. \& West, J. The genetic mosaic suggests a new role for hitchhiking in ecological speciation. Mol. Ecol. 17, 4334-4345 (2008).

25. Feder, J. L. \& Nosil, P. The efficacy of divergence hitchhiking in generating genomic islands during ecological speciation. Evolution 64, 1729-1747 (2010).

26. Lee, Y. et al. Spatiotemporal dynamics of gene flow and hybrid fitness between the $\mathrm{M}$ and $\mathrm{S}$ forms of the malaria mosquito, Anopheles gambiae. Proc. Natl Acad. Sci. USA 110, 19854-19859 (2013).

27. Weetman, D., Wilding, C. S., Steen, K., Pinto, J. \& Donnelly, M. J. Gene flow-dependent genomic divergence between Anopheles gambiae $\mathrm{M}$ and S forms. Mol. Biol. Evol. 29, 279-291 (2012).

28. Reidenbach, K. R. et al. Patterns of genomic differentiation between ecologically differentiated $\mathrm{M}$ and S forms of Anopheles gambiae in West and Central Africa. Genome Biol. Evol 4, 1202-1212 (2012).

29. Neafsey, D. E. et al. SNP genotyping defines complex gene-flow boundaries among African malaria vector mosquitoes. Science 330, 514-517 (2010).

30. Weetman, D. et al. Association mapping of insecticide resistance in wild Anopheles gambiae populations: major variants identified in a low-linkage disequilbrium genome. PLoS ONE 5, e13140 (2010).

31. White, B. J., Cheng, C., Simard, F., Costantini, C. \& Besansky, N. J. Genetic association of physically unlinked islands of genomic divergence in incipient species of Anopheles gambiae. Mol. Ecol. 19, 925-939 (2010).

32. Denholm, I., Devine, G. J. \& Williamson, M. S. Insecticide resistance on the move. Science 297, 2222-2223 (2002).

33. Lynd, A. et al. Field, genetic, and modeling approaches show strong positive selection acting upon an insecticide resistance mutation in Anopheles gambiae s.s. Mol. Biol. Evol. 27, 1117-1125 (2010).

34. Jones, C. M. et al. Footprints of positive selection associated with a mutation (N1575Y) in the voltage-gated sodium channel of Anopheles gambiae. Proc. Natl Acad. Sci. USA 109, 6614-6619 (2012).

35. Davies, T. G. E., Field, L. M., Usherwood, P. N. R. \& Williamson, M. S. DDT, pyrethrins, pyrethroids and insect sodium channels. IUBMB Life 59, 151-162 (2007)

36. Weill, M. et al. The $k d r$ mutation occurs in the Mopti form of Anopheles gambiae s.s. through introgression. Insect Mol. Biol. 9, 451-455 (2000).

37. Dabiré, K. R. et al. Distribution of pyrethroid and DDT resistance and the L1014F $k d r$ mutation in Anopheles gambiae s.l. from Burkina Faso (West Africa). Trans. R. Soc. Trop. Med. Hyg. 103, 1113-1120 (2009).

38. Yawson, A. E., McCall, P. J., Wilson, M. D. \& Donnelly, M. J. Species abundance and insecticide resistance of Anopheles gambiae in selected areas of Ghana and Burkina Faso. Med. Vet. Entomol. 18, 372-377 (2004).

39. Yawson, A. E., Weetman, D., Wilson, M. D. \& Donnelly, M. J. Ecological zones rather than molecular forms predict genetic differentiation in the malaria vector Anopheles gambiae s.s. in Ghana. Genetics 175, 751-761 (2007).

40. Feder, J. L., Egan, S. P. \& Nosil, P. The genomics of speciation-with-gene-flow. Trends Genet. 28, 342-350 (2012).

41. Andrew, R. L. \& Rieseberg, L. H. Divergence is focused on few genomic regions early in speciation: incipient speciation of sunflower ecotypes. Evolution 67, 2468-2482 (2013).

42. Stump, A. D. et al. Centromere-proximal differentiation and speciation in Anopheles gambiae. Proc. Natl Acad. Sci. USA 102, 15930-15935 (2005).
43. Pombi, M., Stump, A. D., Della Torre, A. \& Besansky, N. J. Variation in recombination rate across the $\mathrm{X}$ chromosome of Anopheles gambiae. Am. J. Trop. Med. Hyg. 75, 901-903 (2006).

44. Takahata, N. \& Nei, M. Gene genealogy and variance of interpopulational nucleotide differences. Genetics 110, 325-344 (1985).

45. Wakeley, J. The variance of pairwise nucleotide differences in two populations with migration. Theor. Popul. Biol. 49, 39-57 (1996).

46. Tajima, F. Statistical method for testing the neutral mutation hypothesis by DNA polymorphism. Genetics 123, 585-595 (1989).

47. Chevin, L. M., Billiard, S. \& Hospital, F. Hitchhiking both ways: effect of two interfering selective sweeps on linked neutral variation. Genetics 180, 301-316 (2008).

48. Etang, J. Polymorphism of intron-1 in the voltage-gated sodium channel gene of Anopheles gambiae s.s. populations from Cameroon with emphasis on insecticide knockdown resistance mutations. Mol. Ecol. 18, 3076-3086.

49. Sharakhova, M. V. Genome mapping and characterization of the Anopheles gambiae heterochromatin. BMC Genomics 11, 459 (2010).

50. Presgraves, D. C. Sex chromosomes and speciation in Drosophila. Trends Genet. 24, 336.

51. Coluzzi, M., Sabatini, A., della Torre, A., Di Deco, M. A. \& Petrarca, V. A polytene chromosome analysis of the Anopheles gambiae species complex. Science 298, 1415-1418.

52. Slotman, M., Della Torre, A. \& Powell, J. R. Female sterility in hybrids between Anopheles gambiae and A. arabiensis, and the causes of Haldane's rule. Evolution. 59, 1016-1026 (2005).

53. Nwakanma, D. C. et al. Breakdown in the process of incipient speciation in Anopheles gambiae. Genetics 193, 1221-1231 (2013).

54. Nosil, P., Funk, D. J. \& Ortiz-Barrientos, D. Divergent selection and heterogeneous genomic divergence. Mol. Ecol. 18, 375-402 (2009).

55. Lawniczak, M. K. N. et al. Widespread divergence between incipient Anopheles gambiae species revealed by whole genome sequences. Science 330, 512-514 (2010).

56. Costantini, C. et al. Living at the edge: biogeographic patterns of habitat segregation conform to speciation by niche expansion in Anopheles gambiae. BMC Ecol. 9, 16 (2009).

57. Simard, F. et al. Ecological niche partitioning between Anopheles gambiae molecular forms in Cameroon: the ecological side of speciation. BMC Ecol. 9, 17 (2009).

58. Mitchell, S. N. et al. Identification and validation of a gene causing crossresistance between insecticide classes in Anopheles gambiae from Ghana. Proc. Natl Acad. Sci. USA 109, 6147-6152.

59. Essandoh, J., Yawson, A. E. \& Weetman, D. Acetylcholinesterase (Ace-1) target site mutation $119 \mathrm{~S}$ is strongly diagnostic of Anopheles gambiae carbamate and organophosphate resistance across southern Ghana. Malar. J. 12, 404 (2013).

60. Scott, J. A., Brogdon, W. G. \& Collins, F. H. Identification of single specimens of the Anopheles gambiae complex by the polymerase chain reaction. Am. J. Trop. Med. Hyg. 49, 520-529 (1993)

61. Santolamazza, F. et al. Insertion polymorphisms of SINE200 retrotransposons within speciation islands of Anopheles gambiae molecular forms. Malar. J. 7, 163 (2008).

62. Bass, C. et al. Detection of knockdown resistance $(k d r)$ mutations in Anopheles gambiae: a comparison of two new high-throughput assays with existing methods. Malar. J. 6, 111 (2007)

63. Holt, R. A. et al. The genome sequence of the malaria mosquito Anopheles gambiae. Science 298, 129-149 (2002).

64. Li, H. \& Durbin, R. Fast and accurate short read alignment with BurrowsWheeler transform. Bioinformatics 26, 589-595 (2010).

65. Li, H. et al. The Sequence Alignment/Map Format and SAMtools. Bioinformatics 25, 2078-2079 (2009).

66. Danecek, P. et al. The variant call format and VCFtools. Bioinformatics 27, 2156-2158 (2011).

67. Manske, H. M. \& Kwiatkowski, D. P. LookSeq: a browser-based viewer for deep sequencing data. Genome Res. 19, 2125-2132 (2009).

68. Ellegren, H. et al. The genomic landscape of species divergence in Ficedula flycatchers. Nature 491, 756-760 (2012).

69. R Development Core Team R. R: A Language and environment for statistical Computing. R Foundation for Statistical Computing 1, 409 (2011).

70. Willing, E. M., Dreyer, C. \& Van Oosterhout, C. Estimates of genetic differentiation measured by FST do not necessarily require large sample sizes when using many SNP markers. PloS ONE 7, e42649 (2012).

\section{Acknowledgements}

We thank Dr Robin Fencott for assistance with production of Perl scripts. Sequencing and genotyping support was provided by the Wellcome Trust Sanger Institute and the MalariaGEN resource centre. Additional funding support came from NIAID grant R01AI082734 (D.W. and M.J.D.). C.S.C. was supported by an LSTM Studentship, J.E. by Wellcome Trust MSc Fellowship in Public Health and Tropical Medicine WT094960MA and T.A. by a Sir Henry Wellcome Postdoctoral Fellowship. The funders had no 
role in study design, data collection and analysis, decision to publish, or preparation of the manuscript.

\section{Author contribution}

Project design: D.W., D.K. and M.J.D.; mosquito collection: J.E. and A.E.Y.; genome sequence production: G.M., M.M., B.M. and D.K.; analysis: C.S.C., D.W., S.G.F., M.W., T.A., G.M., J.E. and M.J.D.; manuscript writing: C.S.C., D.W. and M.J.D.

\section{Additional information}

Accession codes: The 15 A. gambiae whole-genome sequences have been deposited in the European Nucleotide Archive database under the accession codes ERS012670 to ERS012684.

Supplementary Information accompanies this paper at http://www.nature.com/ naturecommunications
Competing financial interests: The authors declare no competing financial interests.

Reprints and permission information is available online at http://npg.nature.com/ reprintsandpermissions/

How to cite this article: Clarkson, C.S. et al. Adaptive introgression between Anopheles sibling species eliminates a major genomic island but not reproductive isolation. Nat. Commun. 5:4248 doi: 10.1038/ncomms5248 (2014).

(c) (1)(2) This work is licensed under a Creative Commons Attribution(c) 10 BY $\mathrm{NC}$ SA NonCommercial-ShareAlike 4.0 International License. The images or other third party material in this article are included in the article's Creative Commons license, unless indicated otherwise in the credit line; if the material is not included under the Creative Commons license, users will need to obtain permission from the license holder to reproduce the material. To view a copy of this license, visit http:// creativecommons.org/licenses/by-nc-sa/4.0/ 